\title{
Geometrical control of lattice structures produced by EBM trough chemical etching : investigations at the scale of individual struts
}

\author{
Pierre Lhuissier ${ }^{1,2}$, Charlotte de Formanoir ${ }^{3}$, Guilhem Martin ${ }^{1,2^{*}}$, Rémy Dendievel ${ }^{1,2}$, Stéphane Godet $^{3}$ \\ 1. Université Grenoble Alpes, SIMAP, F-38000 Grenoble, France \\ 2. CNRS, F-38000 Grenoble, France \\ 3. 4MAT, Université Libre de Bruxelles, 50 av. F.D. Roosevelt (CP 165/63), 1050 Bruxelles, Belgium
}

*Corresponding Author: guilhem.martin@simap.grenoble-inp.fr

\begin{abstract}
A major drawback of metal additive manufacturing is the surface roughness of the manufactured components. This is even more critical when complex lattice structures are considered. An octet-truss lattice structure was fabricated by Electron Beam Melting. A chemical post-treatment was applied in order to improve the surface quality. The morphology of individual struts was characterized experimentally by high resolution X-ray tomography after different chemical etching times. The chemical etching treatment was found to be beneficial as it decreases significantly the occurrence of surface defects. The evolution of the elastic mechanical properties with the etching time was determined by FFT computations directly applied to the 3D volume of the struts. A cellular automaton based model was also developed in order to predict the morphological evolution of the as-built strut during etching. The model enables to predict the kinetics of dissolution as well as the evolution of the surface defects and the elastic mechanical properties of the samples. It also enables to determine the required etching time to firstly remove powder particles stuck to the surface and secondly to reduce the plate-pile like defect occurrence.
\end{abstract}

Keywords: Electron Beam Melting; X-ray Tomography; Cellular Automaton; Lattice Structure; Chemical Etching; Mechanical Efficiency 


\section{Introduction}

Unlike most conventional manufacturing processes, additive manufacturing (AM) facilitates the fabrication of complex "near-net shape" parts as well as architectured materials. In particular, lattice structures made of Ti-6Al-4V alloy, which have received a great attention in the literature [1-8], can easily be manufactured by powder bed additive manufacturing (AM) technologies. Among those technologies, Electron Beam Melting (EBM) consists in stacking and melting successive layers of metallic powder, with a high-energy electron beam.

The multiple struts constituting the lattice structure exhibit significant surface irregularities induced by the process. In the context of biomedical applications [7, 9], advantage is taken from this large surface roughness which is known to promote cell growth, see e.g. [10-12]. Lattice structures can also be particularly interesting when weight saving is a crucial requirement such as in the automotive or aeronautic industries. However, contrary to biomedical applications, surface defects turn out to be the most critical limitation for the development and use of lattice structures in automotive and aerospace components. The surface defects are detrimental to static mechanical properties of as-built samples [13, 14]. Indeed, they induce strong stress concentrations that further lead to crack initiation. Both studies reported a beneficial effect on the tensile properties when those surface defects were removed by polishing [13] or machining [14]. Besides, such defects somehow modify the stiffness of the struts because they do not transmit load as a bulk material would. This can be related to the concept of mechanical efficiency introduced by Suard et al. in [15]. Those defects can even be more critical when dealing with dynamic mechanical properties such as fatigue [16]. This as-built surface aspect clearly limits the introduction of lattice structures within parts produced by EBM, when severe requirements in terms of fatigue have to be met. It is therefore important to develop new strategies to reduce those surface defects. Indeed, given the complexity of lattice structures, conventional mechanical post-treatment such as machining cannot be applied. Even classical blasting treatments are not suitable. However, complex mechanical post-treatment such as abrasive flow machining (AFM) [17] or chemical etching, see e.g. [14, 18-22] appear as promising alternatives. A chemical etching procedure was recently developed and successfully applied to lattice structures [23]. This procedure proved (i) to be homogeneous throughout the entire structure, (ii) to significantly increase the stiffness/density ratio (mechanical efficiency) and (iii) to reduce the surface roughness, see [23].

In the present study, the effect of chemical etching on the surface defects of lattice structures produced by EBM was investigated experimentally and numerically. More specifically, the consequences of chemical etching were quantified by a $3 \mathrm{D}$ characterization of the morphology of single struts using high resolution X-ray tomography. The evolution of the morphology of the struts was investigated during the chemical etching procedure. In addition to those experimental results, a 3D cellular automaton based model was developed in order to predict the evolution of the strut morphology during chemical etching. This model is then further discussed when compared to the experimental data. It allows a better understanding of the underlying physical mechanisms driving the chemical dissolution. 


\section{Materials and Methods}

\subsection{Lattice structure production by Electron Beam Melting}

Octet-truss lattice structures were built using an ARCAM A1 EBM machine and Ti$6 \mathrm{Al}-4 \mathrm{~V}$ powder as starting material. The standard "Melt" build theme recommended by ARCAM $\mathrm{AB}^{\circledR}$ for a layer thickness of $50 \mu \mathrm{m}$ was chosen to define the set of melting parameters and scan strategy used during the EBM process. An octet-truss lattice structure $[15,24]$ consisting of 3 unit cells along each direction was produced. The dimensions of the unit cell were $10 \mathrm{~mm}$ x $10 \mathrm{~mm} \times 10 \mathrm{~mm}$. The CAD strut diameter was chosen to be $1.66 \mathrm{~mm}$, resulting in a relative density of $28.5 \%$, measured using standard Archimedes' method. In order to avoid the use of supports and to facilitate the removal of the lattice structures from the build start plate, the lattices were manufactured on an edge, with a $45^{\circ}$ angle with respect to the build start plate. It means that the as-built octet-truss structure consists of struts oriented vertically, horizontally and at $45^{\circ}$ with respect to the build direction.

\subsection{X-ray tomography and chemical etching}

Vertical and horizontal single struts were cut from the as-built lattice structures and then glued on a ceramic holder for the 3D characterization by X-ray microtomography using a Easytom-XL lab tomograph (RX-solution $\left.{ }^{\circledR}\right)$. The individual struts were characterized over a length of about $2.2 \mathrm{~mm}$. The X-ray source used in this study is a Hamamatsu L10711 transmission tube with a $\mathrm{LaB}_{6}$ filament. The detector is a Princeton Instruments Quad-RO 4320 equipped with a $150 \mu \mathrm{m}$ semi-structured CsI scintillator. The acquisition and reconstruction parameters are summarized in Table 1. Volumes were reconstructed using standard filtered back-projection with the software $\mathrm{X}$-act by RX-solution ${ }^{\circledR}$.

\begin{tabular}{cc}
\hline Tube Voltage & $70 \mathrm{kV}$ \\
Target Current & $30 \mu \mathrm{A}$ \\
Filter & $0.5 \mathrm{~mm} \mathrm{Cu}$ \\
\hline Exposure Time & $2.5 \mathrm{~s}$ per projection \\
Number of Projections per Scan & 576 \\
Scan Range & $360^{\circ}$ \\
\hline Voxel Size & $2.5 \mu \mathrm{m}^{3}$ \\
Volume Size (Vertical Strut) & $750 \times 750 \times 882$ Voxels \\
Volume Size (Horizontal Strut) & $750 \times 950 \times 882$ Voxels \\
\hline
\end{tabular}

Table 1. X-ray tomography acquisition and reconstruction parameters.

In order to decrease the surface defects of the as-built struts, a post-process chemical treatment was applied. Between each scan, the single strut was dipped at room temperature in an etchant consisting of $4 \mathrm{~mL} \mathrm{HF} \mathrm{(48 \% ),} 13 \mathrm{~mL} \mathrm{HNO}_{3}(70 \%)$ and $100 \mathrm{~mL}$ of water without being removed from the sample holder. Each strut was placed vertically in a beaker with 100 $\mathrm{mL}$ of etching solution. A fresh etching solution was prepared for each strut: one for the 
vertical strut and a second one for the horizontal strut. Each etching step was immediately followed by extensive water rinsing so as to eliminate any trace of acids. After water rinsing, the strut was replaced at the same position in the laboratory tomograph.

3D images of the struts were acquired in the as-built conditions, i.e. before any etching and respectively after 10, 20,30 and $60 \mathrm{~min}$ of chemical etching. Due to poor bonding of the sample on the holder, the initial state (as-built, before etching) of the horizontal strut is missing. Indeed, the sample moved during the X-ray tomography scan making the reconstruction of the corresponding 3D volume impossible. The problem was discovered too late to scan again the sample in its as-built configuration. Thus, for the horizontal as-built strut, the reference state is the strut that was the one chemically etched for $10 \mathrm{~min}$.

3D images were first filtered with a 3D median filter with a radius of 3 voxels. Since the volumes only contain two phases, namely the material and the pores, and that they can easily be discriminated based on grayscale histograms, images were binarized using an automatic threshold. Note that this thresholding step was not very sensitive to the algorithm selected. The strut volume at step $n$, denoted $V_{t_{n}}$ is defined as the sum of bulk material voxels after an etching time $t_{n}$. All the pores were labeled and their respective size and position recorded. Some of these pores were tracked from one scan to another in order to accurately stitch the different scans performed on the same strut.

\subsection{Data Processing}

\subsubsection{Dissolution Kinetic}

The etching kinetic was measured globally and locally. The overall etched volume fraction was defined as the volume difference between two successive etching steps. The values were normalized by the initial volume $\left(V_{t_{0}}\right)$, i.e. the volume of the as-built strut as indicated in equation (1):

$$
f_{V}^{\text {etched }}=\left(V_{t_{n-1}}-V_{t_{n}}\right) / V_{t_{0}} \text {. }
$$

This volume fraction, denoted $f_{V}^{\text {etched }}$, is then plotted as a function of time.

A specific procedure was developed in order to measure the local etching rate. Once a voxel was determined as belonging to the border at time $t_{n}$, its position was somehow tracked back on the scan at time $t_{n-1}$. The local etching rate of that voxel was then estimated based on the minimum distance to the surface at time $t_{n-1}$. The distances initially given in voxels were then converted into rate using the voxel size and the time increment (equal to the difference between $t_{n}$ and $\left.t_{n-1}\right)$. 


\subsubsection{Roughness}

The profile of the surface irregularities was extracted along the generatrices of the 3D volume of individual struts. The analyzed length of the individual strut was typically about $2.2 \mathrm{~mm}$. By steps of $10^{\circ}, 36$ profiles were analyzed for each strut. For each profile, the arithmetic roughness $\left(R_{a}\right)$ as well as the maximum height of the profile $\left(R_{t}\right)$ were computed over the length of the strut. More details regarding the roughness calculations can be found in [15].

\subsubsection{Mechanical efficiency : concepts of equivalent diameters}

3D images were also used to compute the mechanical elastic response of the single struts in the as-built conditions as well as after different chemical etching times. Note that in an octet-truss structure, all the struts are only submitted to tension/compression loading. Therefore, the mechanical efficiency of the lattice structure can be estimated based on the tension/compression behavior of an individual strut. Note that this methodology is not perfect since the complexity of the stress state in the vicinity of the strut intersections (nodes of the lattice) is probably not fully captured. As-built or etched strut was assimilated to a perfect cylinder with an equivalent diameter corresponding to a given property: volume or stiffness in the present case. As a result, the concept of equivalent cylinder introduced in [15] was used to express the strut volume and stiffness.

First, a volumetric equivalent cylinder can be defined as a perfect cylinder having the same length and the exact same volume as the strut. The diameter of the strut is denoted $D_{e q}^{\text {vol }}$. Note that this cylinder is an upper bound for the stiffness of the strut for a tension/compression loading since it assumes that all the material is efficiently used to transmit axial stresses.

Secondly, a stiffness equivalent diameter can be defined based on a numerical simulation performed directly on the voxelized image resulting from the X-ray tomography characterization. The idea is to compute the effective stiffness of the strut based on 3D images for a tension/compression loading. This numerical analysis relies on a Fast Fourier Transform (FFT) analysis using the CraFT software [25] as described in [15]. It is based on the computation of the elastic stiffness of a two phase image and the resolution of the stressstrain relationship in the Fourier space. The image used as an input consists of two phases: bulk Ti-6Al-4V alloy and air. In this two phases computation, air was given a substantial stiffness in order to limit the stiffness contrast. Indeed, the method cannot converge for very large stiffness contrast. Nevertheless, this contrast was chosen large enough (1000) to avoid any significant effect on the results [15]. The parameters for the stiffness calculations are given in Table 2. Computations were performed on binned volume in order to reduce the computation time. The outputs of the FFT simulation are the macroscopic stress and strain along every direction. The stiffness along the strut axis direction was calculated from the stress and strain along this direction. From those numerical simulations, the effective stiffness of the strut can be determined. Thus a mechanical equivalent diameter, denoted as $D_{e q}^{\text {stiffness }}$ can 
be defined and corresponds to a perfect cylinder having a stiffness equal to the strut stiffness computed numerically.

Those two equivalent diameters are related to the concept of mechanical efficiency. In other words, those concepts allow differentiating the material that transmits loads from the

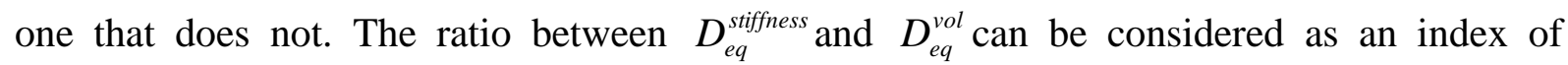
mechanical efficiency. This index ranges between 0 and 1 . When the material is inefficiently distributed $D_{e q}^{v o l}$ is much larger than $D_{e q}^{s t i f f n e s s}$ and the index is close to zero. When almost all the material is carrying load, the index is close to 1 . A perfect cylindrical strut has an index value equal to 1 .

\begin{tabular}{cc}
\hline Young's modulus of the constitutive material (Ti-6Al-4V) & $114 \mathrm{GPa}$ \\
Poisson ratio of the constitutive material (Ti-6Al-4V) & 0.345 \\
Stiffness contrast & $10^{3}$ \\
\hline Convergence criterion on stress & $10^{-3}$ \\
\hline
\end{tabular}

Table 2. Parameters for the FFT calculations of the stiffness using CraFT. 


\section{Cellular Automaton based Model}

The goal is to develop a model that would enable (i) to predict the evolution of the strut morphology with the etching time and (ii) to provide an insight into the underlying physical mechanisms driving the chemical dissolution.

\subsection{Description of the model}

For each voxel of bulk material, the applied model will select if the voxel remains a bulk material one or if it is dissolved chemically. The criterion is simply based on a critical volume of etchant contained in the vicinity of the voxel of interest. A cellular automaton based model was applied to the 3D images of the initial strut. In those calculations, the 3D images are considered as consisting of two phases: the bulk material, and the chemical solution. A 2D schematic illustrating the cellular automaton based model is provided in Figure 1a. Note that the calculations are performed in 3D. At each iteration and for each voxel, the local density of etchant $\rho_{\text {etchant }}$ inside a sphere of radius $R$ is computed. The local density of etchant $\rho_{\text {etchant }}$ is calculated as the ratio of the volume of etchant over the volume of the sphere of radius $R: \rho_{\text {etchant }}=V_{\text {etchant }} / V_{\text {sphere, }}$. It is then compared to a critical density of etchant denoted $\rho_{c}$. If the local density of etchant $\rho_{\text {etchant }}$ is larger than the critical one $\rho_{c}$ then the voxel is converted into chemical solution, otherwise the voxel remains a bulk material voxel, see Figure 1b. Therefore, two parameters have to be adjusted: the local radius denoted $R$ and the critical density of etchant $\rho_{c}$. Given the relatively small volume of the individual strut compared to the volume of the etching solution, the acid concentrationwas assumed to remain constant with the etching time. This particular point is discussed in section 5.1.

The model relies on successive iterations performed on the 3D images. The algorithm was implemented in CUDA (parallel computing platform) on a GPU (Graphic Processing Unit) in order to use the high degree of parallelism of the 3D data. 


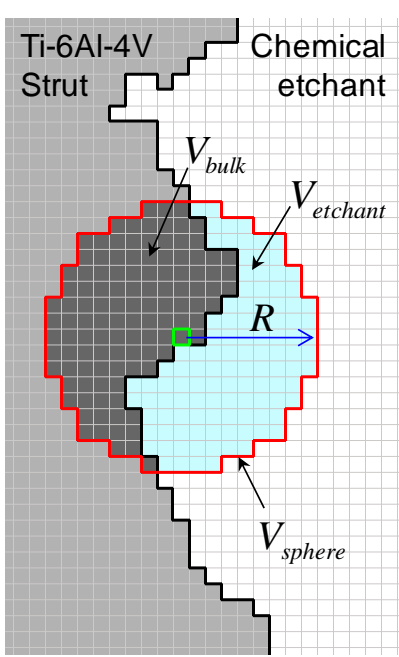

(a)

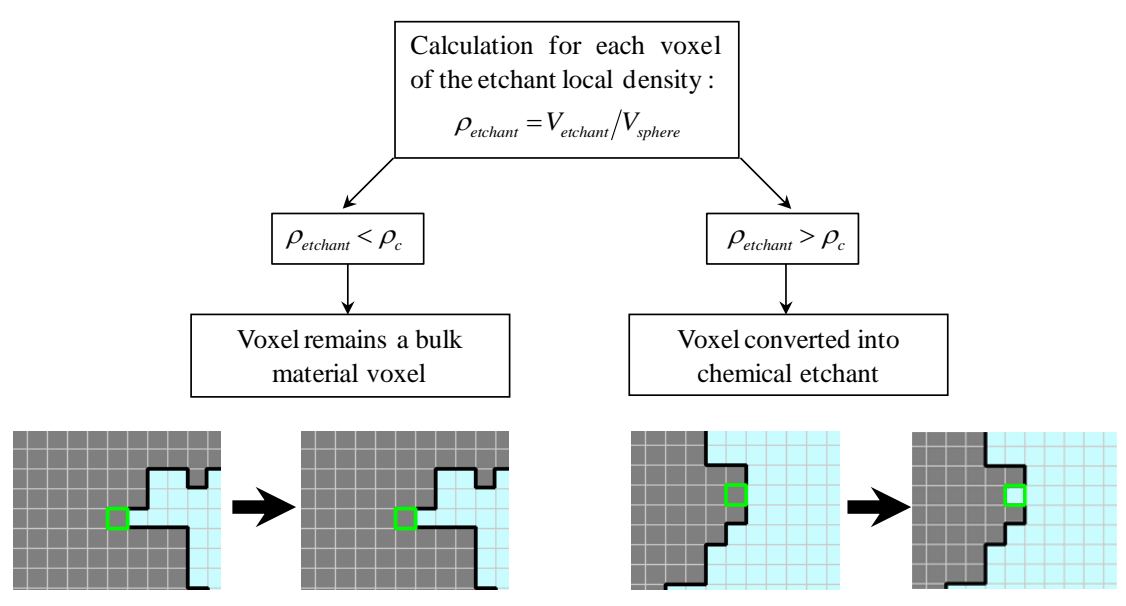

(b)

Figure 1. (a) 2D Schematic of the cellular automaton. Note that the developed cellular automaton has been used in 3D. (b) Summary of the procedure used by the cellular automaton. The voxel of interest is highlighted in green.

\subsection{Adjustment of the model}

The parameters of the model, namely $R$ and $\rho_{c}$, were adjusted so as to reproduce as closely as possible the experimental results acquired for the vertical strut. Note that in addition to $R$ and $\rho_{c}$, the model has another free parameter: the physical time increment $\Delta t$ corresponding to one iteration. Thus a global optimization was conducted on the three parameters: $R, \rho_{c}$ and $\Delta t$. Note that the 3 parameters do not have the same status: for each couple $\left(R, \rho_{c}\right)$ a simulation must be conducted, while $\Delta t$ is just a scaling parameter applied on the results. The model is applied to the initial 3D image as-characterized by X-ray tomography. It means that the model allows the evolution of the whole strut to be described during chemical etching. For each set of guess parameters $\left(R\right.$ and $\left.\rho_{c}\right)$ a simulation with 100 iterations was performed. Then the physical time corresponding to one iteration $\Delta t$ is optimized in order to minimize the deviation from the experimental data. The deviation from the experimental data is defined as follows. For a given time increment $\Delta t$ and a given experimental etching time $t_{i}\left(t_{i}=10,20,30\right.$ or $\left.60 \mathrm{~min}\right)$ a partial error is computed by comparing the experimental data at $t_{i}$ with the simulated data at the corresponding iteration. This partial error is the sum of the voxels that have been erroneously etched by the model and the voxels that should have been etched according to the model but have not. Finally the global error for a given time increment $\Delta t$ is computed as the sum of the partial errors for the four etching times (10, 20, 30 and $60 \mathrm{~min}$ ). The optimal parameters are reported in Table 3. It is important to note that the model was adjusted only using the experimental results acquired for the vertical strut. The optimal set of parameters might not be unique. However, the same set of parameters was also used as input to correctly describe the horizontal strut evolution as seen later in section 4 . 


\begin{tabular}{cc}
\hline Local radius, $R$ & 7 voxels \\
Threshold etchant density, $\rho_{c}$ & 0.42 \\
Physical time corresponding to one iteration, $\Delta t$ & $0.91 \mathrm{~min}$ \\
\hline
\end{tabular}

Table 3. Optimal parameters of the model based on a comparison with the experimental data of the vertical strut.

\section{Results}

\subsection{Identification and classification of surface defects}

Surface defects inherited from the EBM process can be observed in as-built struts, especially on struts built vertically, Figure 2. Those surface defects can be classified into two categories:

- Type I: powder particles not fully melted and stuck to the strut;

- Type II: plate-pile like stacking irregularity.

The plate-pile like stacking defect was already reported $[15,26]$ and discussed in details by Korner et al. in [26]. This defect manifests itself as protrusions. The 2D schematic crosssection shown in Figure 2a enables to identify clearly both types of defects. They can be observed on the SEM micrograph of an as-built vertical strut shown in Figure $\mathbf{2 b}$.

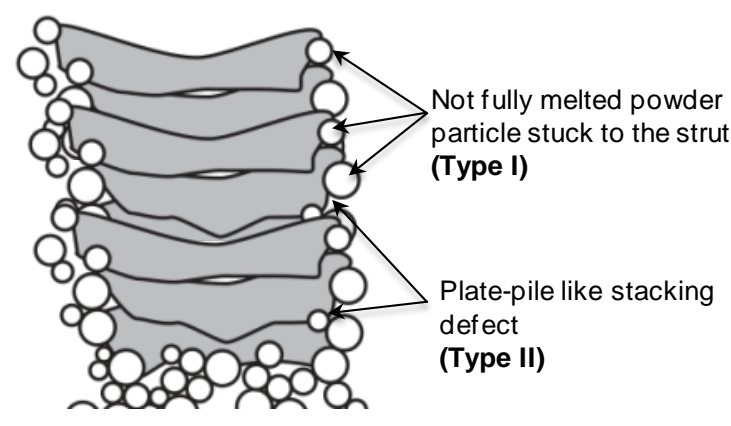

(a)

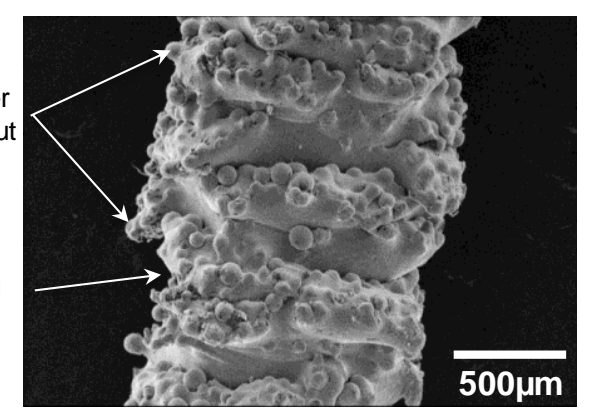

(b)

Figure 2. (a) Schematic illustrating both types of surface defects observed on as-built strut, inspired from [26] and (b) SEM micrograph of a vertical single strut showing both types of defects. 


\subsection{Overall behavior}

The morphological evolutions of the vertical and horizontal struts with the etching time are shown on 2D cross-sections in Figure 3a and Figure 3b. There is a significant difference in morphology between the vertical and horizontal as-built struts. This has already been observed and attributed to a difference in local heat transfer [15].

As the etching time increases, the surface defects are clearly reduced for both vertically and horizontally built struts. This is further evidenced by the difference between the as-built surface of the vertical strut (red profile in Figure 3a) and the etched surface after different etching times. For instance, the surface profile of the 30 min-etched vertical strut (yellow profile in Figure 3a) is clearly much smoother than the one of the as-built strut. In the first 20 minutes of etching time, Type I surface defects, i.e. powder particles not fully melted and stuck to the strut, are preferentially etched, see Figure 3a. Between 20 and 60 minutes of etching, the plate-pile like stacking defects (Type II) appears to be slowly smoothed.

The volume fraction of material dissolved chemically during etching is reported in Figure 4 for both vertical and horizontal struts as a function of etching time. The overall etching rate, which is the slope of the curves in Figure 4, is higher in the first 10 minutes of etching. For longer etching times, it tends to become constant (quasi linear trend) because the strut morphology tends to a perfect cylinder. After 20 min of etching time, about $15 \%$ of the volume of the vertical as-built strut has been chemically dissolved and after 60 min about one third of the as-built volume has disappeared.

The optimized cellular automaton enables to predict the kinetic of the chemical dissolution. It has to be noticed that the two struts behave very similarly in terms of global etching rates. It is even more noticeable that the model is able to predict the dissolution kinetic of the horizontal strut, although it was adjusted and optimized based only on the experimental results derived for the vertical strut. 

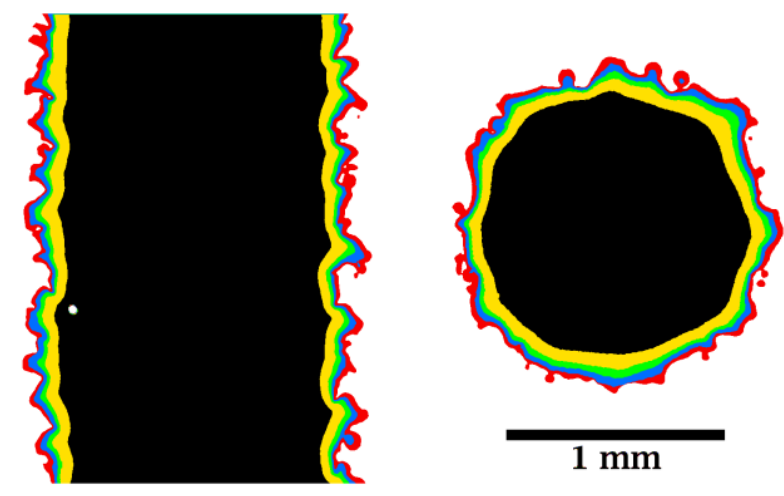

$1 \mathrm{~mm}$

(a)
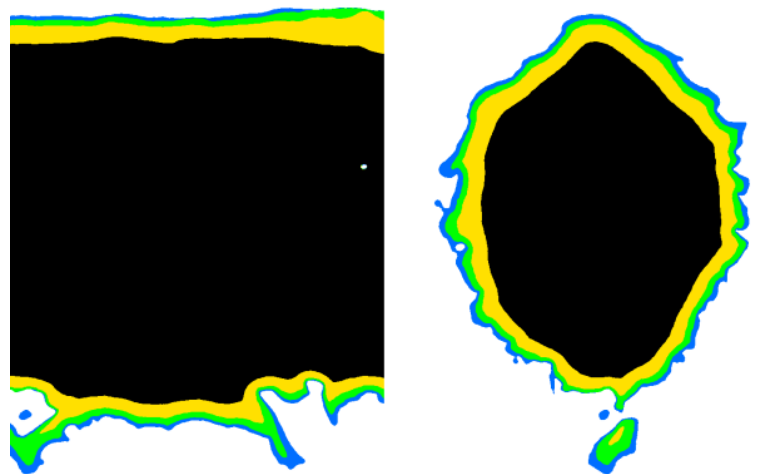

(b)

Figure 3. 2D sections of the vertical strut (a) and horizontal strut (b). The color refers to the bulk material that has been dissolved through chemical etching after each time increment (as-built in red, 10 min-etched in blue, 20 min-etched in green, 30 min-etched in yellow, and 60 min-etched in black). A color version is available on the online version of the paper. Note that the as-built conditions for the horizontal strut is missing due to poor bonding of the sample on the holder as explained in section 2.2.

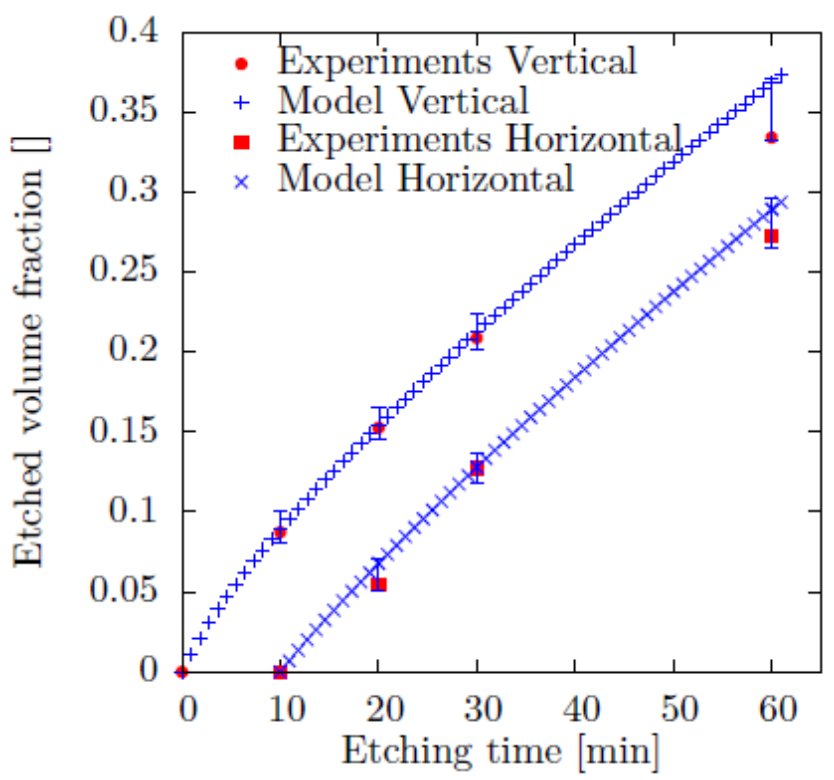

Figure 4. Volume fraction of etched bulk material as a function of etching time: experimental data in red and numerical model in blue. Error bars represent overetched and underetched volumes of the numerical model. Note that the as-built horizontal strut data are missing as explained in section 2.2. 
The local etching rate, as defined in 2.3 , was measured experimentally based on the 3D images acquired by X-ray tomography. The results are presented for the vertical strut in Figure 5a where the color code corresponds to the local magnitude of the etching rate. The etching rate was found to be very heterogeneous during the first 20 minutes of etching. Some regions are quickly etched while others are almost unaffected. The highest local etching rates, around $7-8 \mu \mathrm{m} / \mathrm{min}$ were measured for the powder particles stuck to the melt pool (Type I defect). As the etching time increases, the etching rate becomes more and more homogeneous and reaches 2-3 $\mu \mathrm{m} / \mathrm{min}$. After 20 minutes of etching, the powder particles are almost entirely dissolved and the surface profile becomes smoother. This observation is consistent with the observations made on the 2D cross sections shown in Figure 3. Figure 5b presents the local etching rate profile superimposed on the simulated data. The cellular automaton enables to predict the overall behavior, and it turns out that it also provides a very good description of the local behavior. In other words, the local etching rates are also rather well predicted by the cellular automaton based modeling. Not only does the model predict the regions that are preferentially etched but it also provides a good estimation of the local etching rates. 


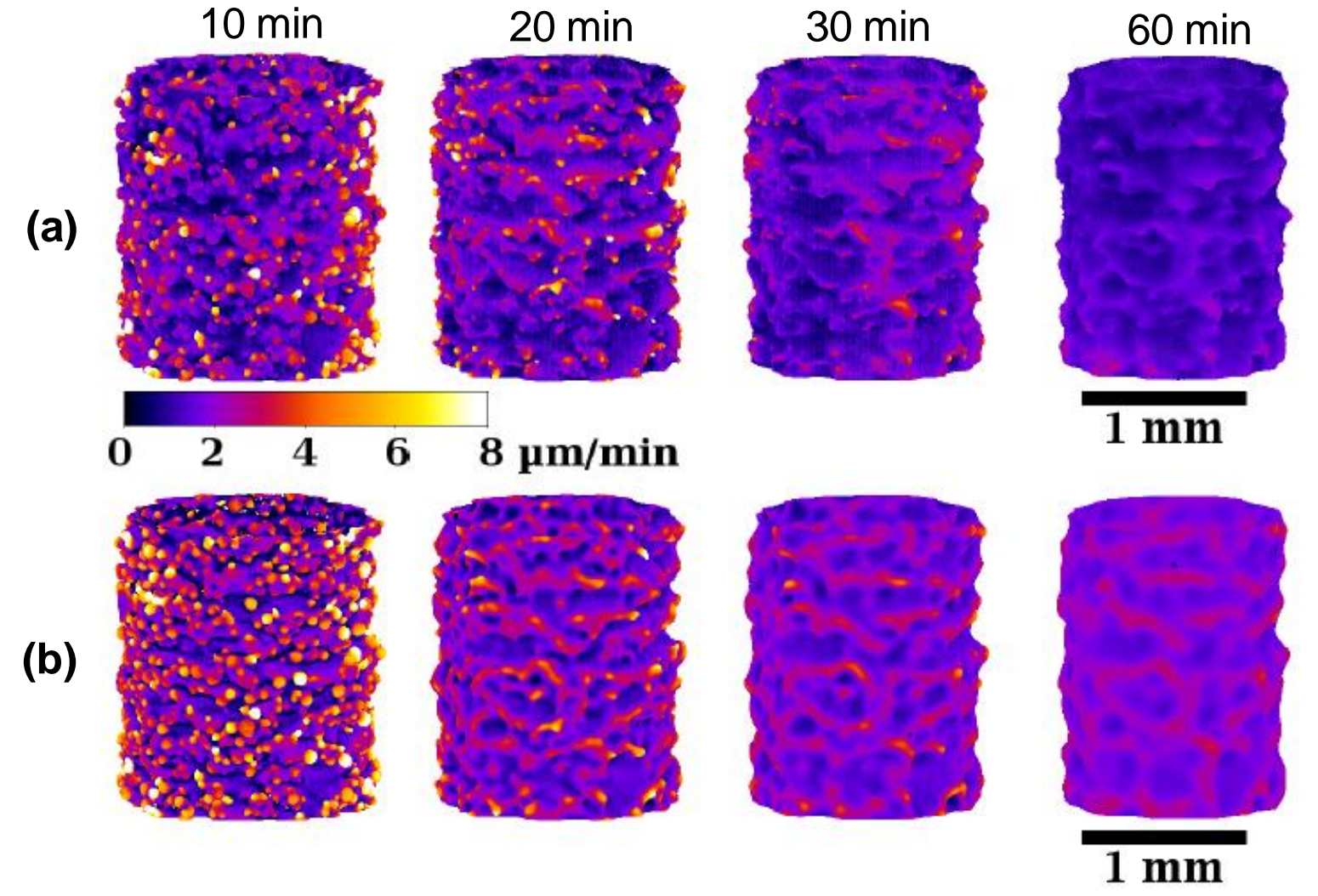

Figure 5. 3D views of the vertical etched strut after 10, 20, 30 and 60 min etching times. (a) Experimental results and (b) predictions of the cellular automaton based modeling. The color bar represents the local etching rate.

\subsection{Roughness}

The roughness can be described qualitatively at two scales corresponding to the two categories of surface defects mentioned previously, i.e. Type I and Type II. Both types of defects increase the surface roughness significantly. The surface roughness was quantitatively analyzed and the evolution of the roughness with the etching time is plotted in Figure 6a and Figure $\mathbf{6 b}$ for the vertical and horizontal strut, respectively. For both orientations, the arithmetic roughness $\left(R_{a}\right)$ as well as the maximum roughness $\left(R_{t}\right)$ can be substantially reduced through chemical etching. For instance, the as-built vertical strut exhibits an average roughness of $\sim 35 \mu \mathrm{m}$ which goes down to $\sim 20 \mu \mathrm{m}$ after 60 minutes of chemical etching.

The roughness decreases significantly during the first 20 minutes of etching and it decreases more slowly between 20 and $60 \mathrm{~min}$. These two regimes correspond to the previous observations stating that powder particles stuck to the melt pool were chemically dissolved rather fast, as it can be seen in Figure 5. Again the cellular automaton based model provides results consistent with the experimental data. Note that the discrepancies are larger for the horizontal strut. This can be explained by the particular morphology of the horizontal strut. Indeed, a clear asymmetry between top-facing and down-facing surfaces is observed making difficult an overall roughness to be estimated, see Figure $\mathbf{3 b}$. 


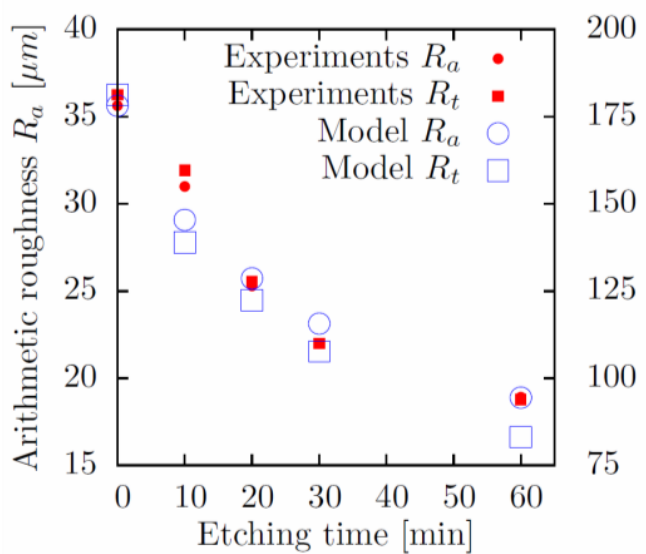

(a)

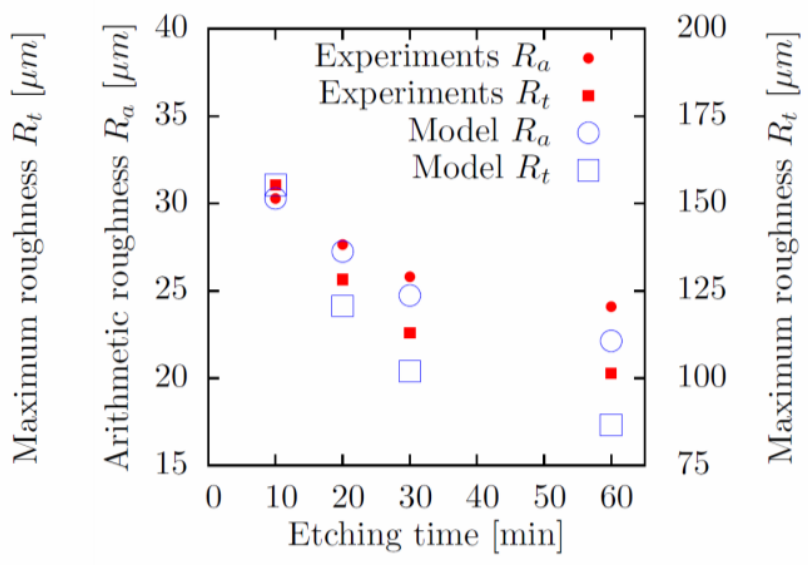

(b)

Figure 6. Evolution of the arithmetic roughness $R_{a}$ (circles) and maximum roughness $R_{t}$ (squares) with the etching time: (a) vertical strut and (b) horizontal strut. Experimental results in red and simulation data in blue.

\subsection{Mechanical efficiency}

Both concepts of mechanically equivalent diameters, i.e. volumetric equivalent diameter $\left(D_{e q}^{v o l}\right)$ and numerically equivalent diameter $\left(D_{e q}^{\text {siffress }}\right)$ were used to quantify the mechanical efficiency of the struts as the etching time increases. The difference between equivalent diameters is significant in the as-built conditions but becomes smaller as the etching time increases, as it can be seen in Figure 7a and Figure 7c. This is verified on both strut orientations. In other words, it means that part of the material present in the surface defects does not transmit mechanical loads ( $D_{e q}^{v o l}$ is larger than $\left.D_{e q}^{\text {siffress }}\right)$. When increasing the etching time, the surface defects are reduced through chemical dissolution and therefore a higher fraction of material is mechanically efficient. Indeed, as shown in Figure $\mathbf{7 b}$ and Figure 7d, $D_{e q}^{\text {vol }}$ is closer to $D_{e q}^{\text {siffress }}$ when the etching time increases.

The index of mechanical efficiency, defined as $D_{e q}^{\text {siffress }} / D_{e q}^{v o l}$ is about 0.942 for the asbuilt vertical strut. After one hour of etching, the index of mechanical efficiency reaches almost 0.985 . In other words it means that in the as-built conditions the strut exhibits an overweight of $\sim 11 \%$ regarding its mechanical properties. This overweight was computed as $\left(V_{e q}^{v o l}-V_{e q}^{\text {stiffress }}\right) / V_{e q}^{v o l}=1-\left(D_{e q}^{\text {siffress }} / D_{e q}^{v o l}\right)^{2}$. This overweight decreases to less than $3 \%$ after one hour of etching. 


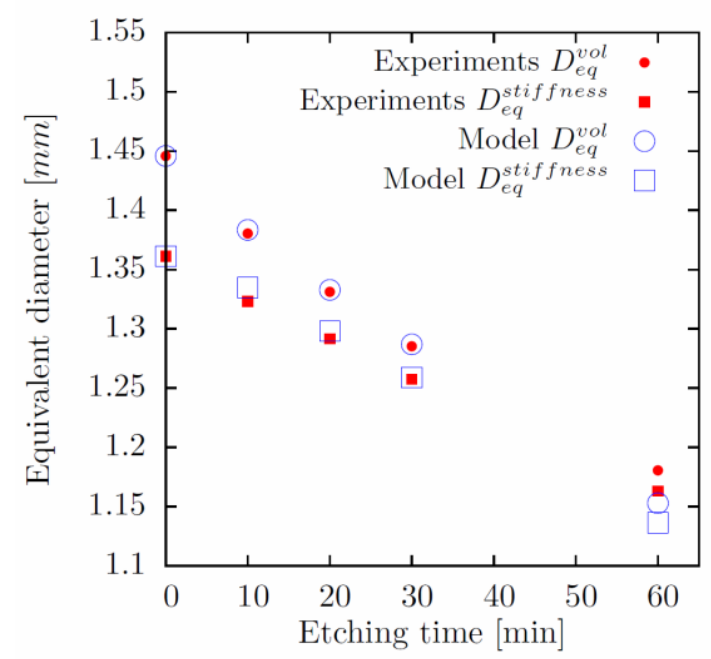

(a)

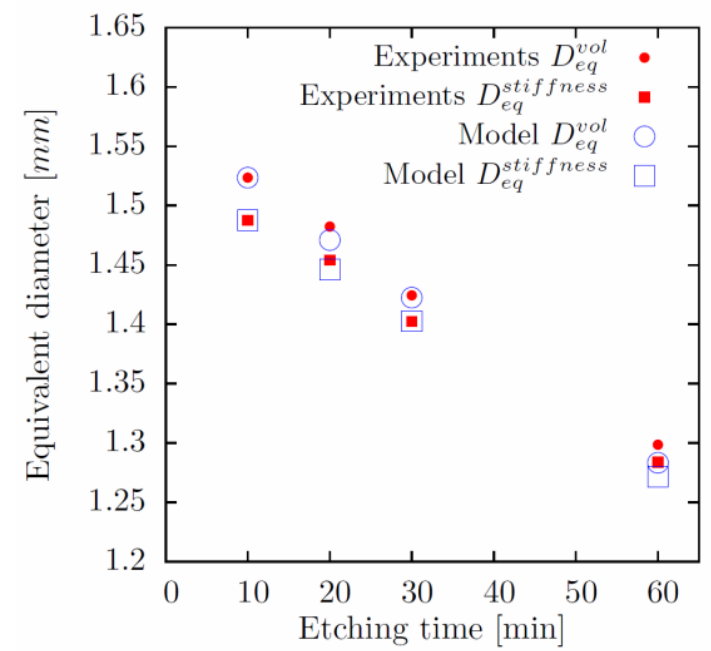

(c)

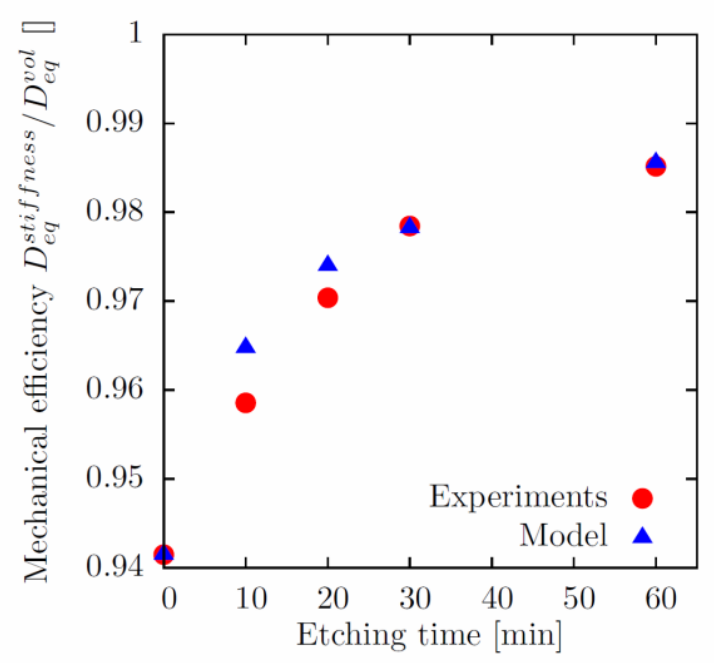

(b)

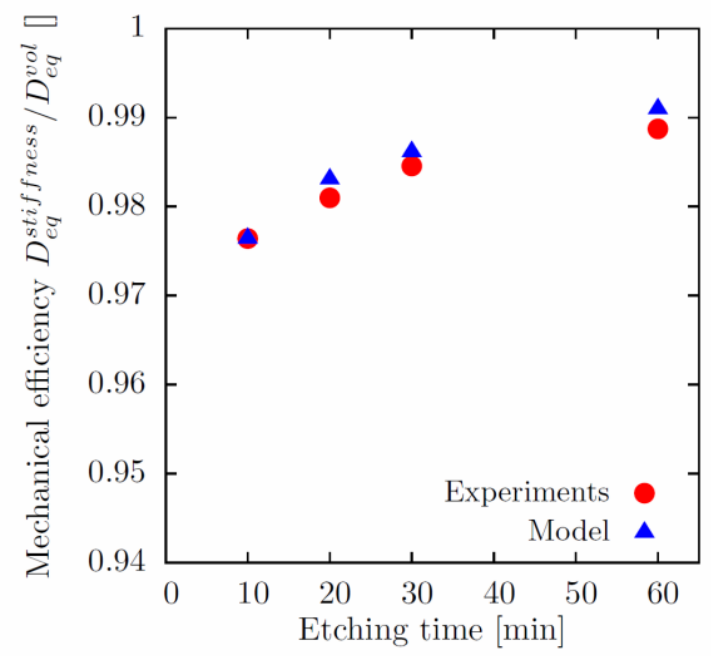

(d)

Figure 7. Evolution of the volumetric equivalent diameter, $D_{e q}^{v o l}$ and the mechanical equivalent diameter, $D_{e q}^{\text {stiffness }}$ with the etching time for (a) the vertical strut and (c) horizontal strut. Evolution of the index of mechanical efficiency with the etching time for (b) vertical strut and (d) horizontal strut. 


\section{Discussion}

\subsection{Possible origin of the discrepancies between the experimental results and the predictions of the cellular automaton}

The 3D characterization of single struts using high resolution X-ray tomography after different chemical etching times enables to evaluate if such treatment would be beneficial to improve the surface of lattice structures produced by additive manufacturing. In particular, several relevant parameters were quantified to evaluate the effect of chemical etching:

(i) the kinetic of dissolution;

(ii) the surface roughness

(iii) the mechanical efficiency, i.e. the fraction of material that transmits loads.

The simple cellular automaton modeling method presented here provides a good description of the morphological evolution of the individual struts. This was assessed by comparing the three parameters mentioned previously with those determined experimentally. A rather good agreement was achieved between the cellular automaton based model and the experiments. However, some discrepancies between the experiments and the model can still be observed, in particular for a 60 minutes etching time. This discrepancy can be discussed based on two considerations.

First, the chemical reactions leading to the dissolution of the Ti-6Al-4V alloy comes along with an outgassing phenomenon [14]. This was confirmed by the observation of the formation of a gas bubbles around the sample during chemical etching. Some of these gas bubbles might be located in the vicinity of certain surface defects and might lead to a significant decrease of the local density of etchant. The local density of etchant was clearly identified as a critical parameter when one aims at predicting the evolution of the strut morphology during chemical etching. In order to limit that issue small vibrations were manually applied to the sample when dipped into the etching solution to allow the gas bubbles to escape. The frequency of application of those manual vibrations was significantly reduced during the last 30 minutes of etching time. Note that a simple stirring of the etching solution was found not to be as efficient to promote the gas bubbles to escape. As a result, it is suggested that the bubbling can affect the local density of etchant and therefore slightly slows down the kinetic of dissolution, especially when the sample is not submitted to high frequency vibrations.

Secondly, the chemical reactions leading to the dissolution of the Ti-6Al-4V alloy might also affect the local chemical composition of the etchant. Indeed, after relatively long etching time, the chemical solution can achieve a situation where the solution is locally supersaturated and cannot dissolve Ti-6Al-4V alloy anymore unless an efficient stirring is applied. It is thought that this phenomenon might also partly explain the discrepancy between the model and the experiments.

\subsection{Mechanism driving the chemical dissolution}


It is now interesting to study in more details the physical insights that the model can provide regarding the chemical dissolution mechanisms. The cellular automaton relies on a condition based on the local density of etchant in the vicinity of the voxel of interest. This rather simple rule implies that concave or convex surfaces do not behave similarly, see Figure 8. Convex surfaces, corresponding typically to the powder stuck to the strut, are dissolved quicker than concave ones. Indeed, the local density of etchant contained in the sphere surrounding the voxel of interest is larger when the surface exhibits a convex surface compared to when the surface exhibits a concave shape as highlighted in Figure 8. As a direct consequence, the plate-pile like stacking defect starts to be affected by the etchant once the local density of etchant becomes significant, i.e. once the powders stuck to the strut have been removed. It means the plate-pile like stacking defect is not accentuated with the etching time. An illustration of the sequence of chemical dissolution of the different surface defects is provided in Figure 9.

In addition to give relatively good predictions of the strut evolution during chemical etching, the cellular automaton suggests that the physical mechanism behind the chemical dissolution of the surface defects is driven by the local density of etchant. In other words, the chemical dissolution depends significantly on the amount of etchant that is surrounding the surface defect. This observation might be consistent with the diffusion control nature of the chemical etching process. When a small volume of etchant is locally surrounded by a large amount of material, it should tend to chemically saturate, slowing down the local etching rate. On the other hand, a small material protrusion surrounded by a large volume of etching solution should not modify significantly the local concentration of the etchant. These observations are just based on geometrical evolution of the strut during chemical etching. Validating these assumptions would require a full chemical investigation which is not in the scope of the present paper. 


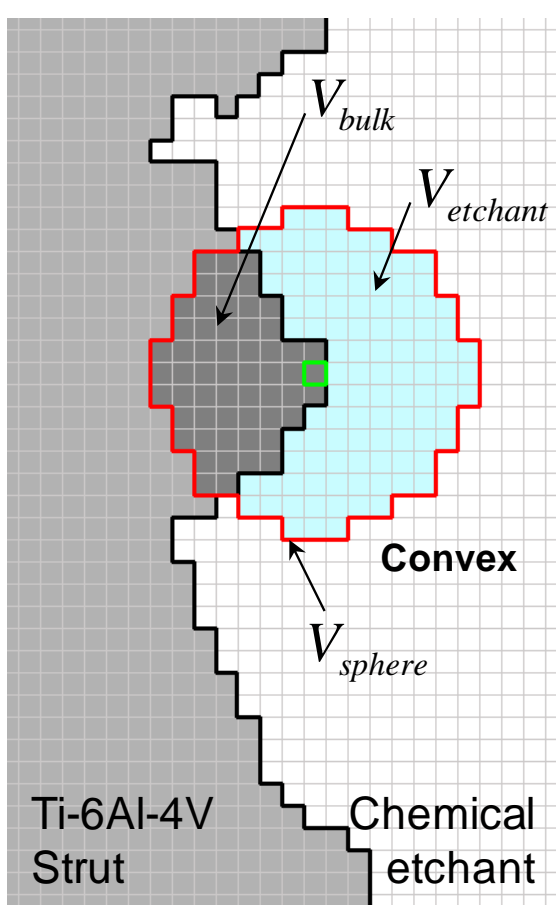

(a)

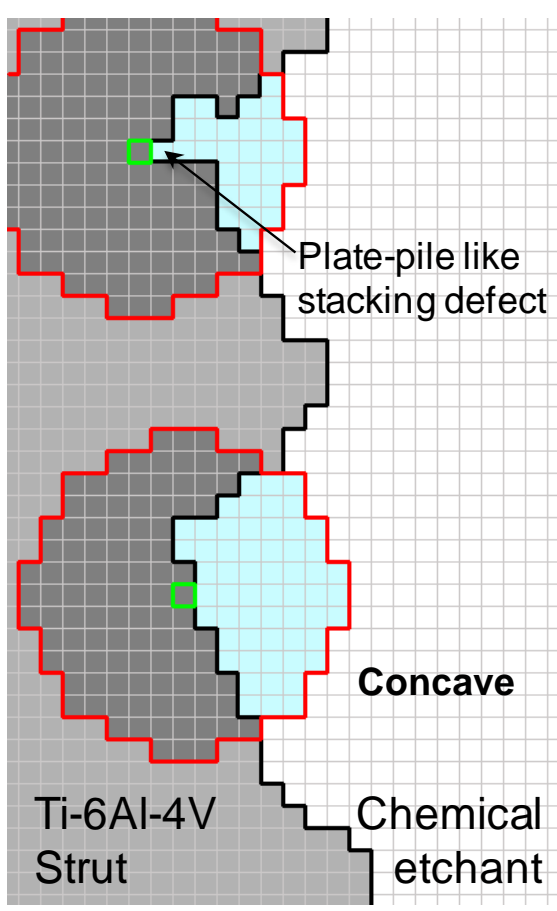

(b)

Figure 8. 2D schematic of the cellular automaton (a) for a voxel located on a convex surface and (b) for a voxel located on a concave surface and at a the tip of a plate-pile like stacking defect. The pixel of interest is highlighted in green.
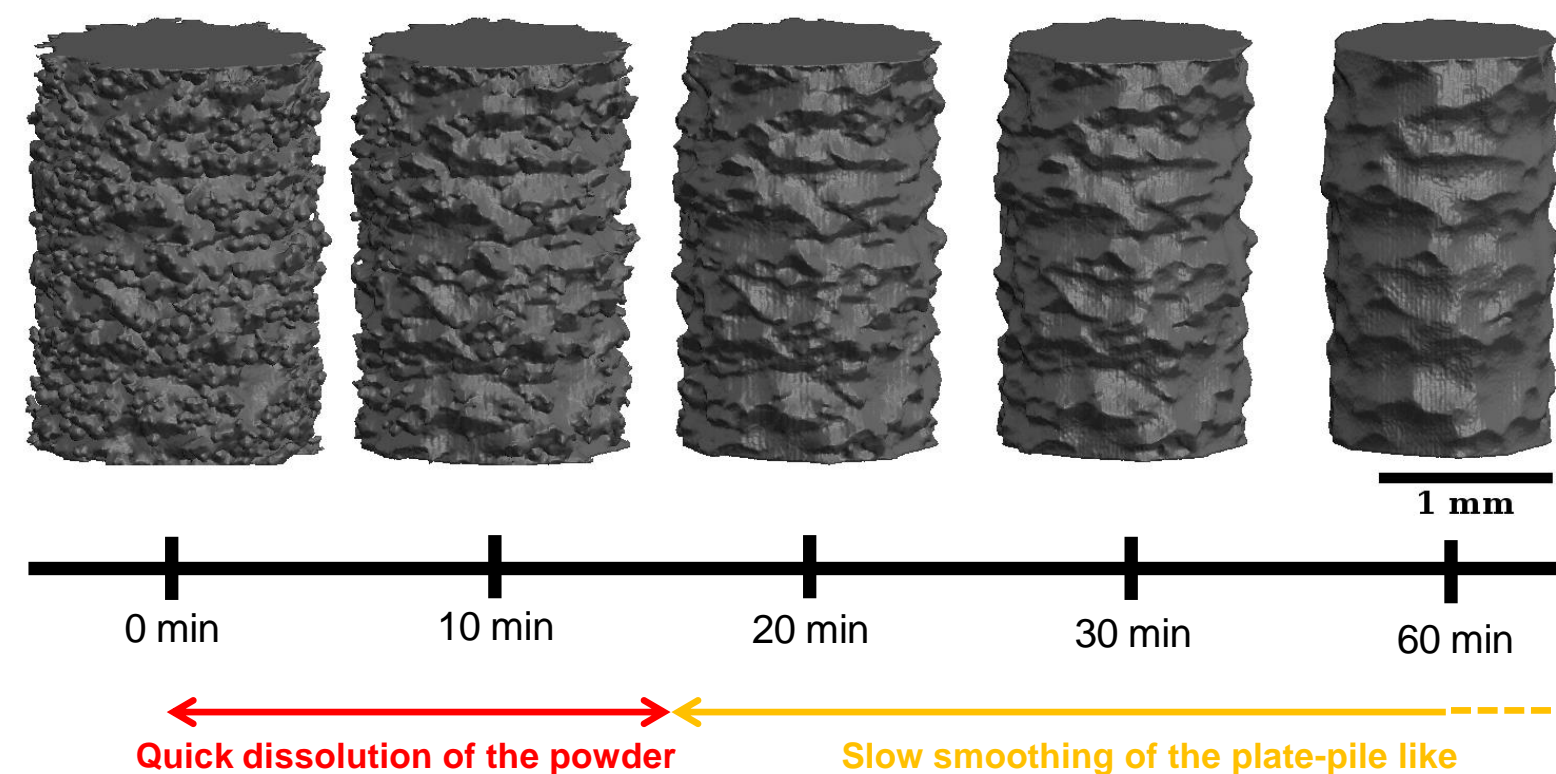
particles stuck to the melt pool 'Type l' surface defect

\section{Slow smoothing of the plate-pile like defect \\ ‘Type II' surface defect}

Figure 9. Sequence of chemical dissolution of the different types of surface defects (experiments): Type I is quickly dissolved in the first 15 minutes while Type II is more slowly dissolved between 15 and 60 min of etching time. 


\section{Conclusion}

The evolution of the strut morphology, the surface roughness as well as the mechanical efficiency of single struts pertaining to lattice structures were quantified by X-ray tomography before and after chemical etching. The present chemical etching post-treatment leads to a substantial and homogeneous reduction of surface defects inherited from the EBM process. The significant decrease in roughness combined to the significant increase of the mechanical efficiency after 30 min-chemical etching results from the removal of a high fraction of surface defects. Such a chemical post-treatment might be considered if one aims at improving the static as well as the dynamic mechanical properties of lattice structures produced by EBM.

A simple cellular automaton model allowed the underlying mechanism driving chemical etching process to be captured and the morphological strut evolution to be predicted. This was verified on both vertical and horizontal struts. Since the local morphological evolutions are well-predicted, global properties such as roughness or mechanical efficiency are also correctly predicted. The low sensitivity of the model to significant morphological changes as the one highlighted between a vertical and an horizontal strut is promising. Indeed, it could be applied to more complex parts such as entire lattice structures where struts junctions evolution during chemical etching is not yet understood. In addition, the range of validity of the model should be investigated in more details for lattice structures exhibiting finer porosity. For instance, bubbling or acid depletion of the etchant should be taken into account.

Further work is required in order to investigate the possible benefit of this chemical etching procedure on the fatigue behavior of lattice structures. This is the aim of an ongoing research. 


\section{Acknowledgements}

This work was performed within the framework of the Center of Excellence of Multifunctional Architectured Materials "CEMAM"n॰AN-10-LABX-44-01 funded by the "Investments for the Future" Program. The authors are also grateful to the F.R.I.A. - F.N.R.S. for financing part of this research.

\section{References}

[1] Heinl P, Rottmair A, Koerner C, Singer RF. Advanced Engineering Materials 2007;9:360.

[2] Heinl P, Koerner C, Singer RF. Advanced Engineering Materials 2008;10:882.

[3] Cheng XY, Li SJ, Murr LE, Zhang ZB, Hao YL, Yang R, Medina F, Wicker RB. Journal of the Mechanical Behavior of Biomedical Materials 2012;16:153.

[4] Gaytan SM, Murr LE, Martinez E, Martinez JL, Machado BI, Ramirez DA, Medina F, Collins S, Wicker RB. Metallurgical and Materials Transactions a-Physical Metallurgy and Materials Science 2010;41A:3216.

[5] Li SJ, Murr LE, Cheng XY, Zhang ZB, Hao YL, Yang R, Medina F, Wicker RB. Acta Materialia 2012;60:793.

[6] Murr LE, Gaytan SM, Medina F, Martinez E, Martinez JL, Hernandez DH, Machado BI, Ramirez DA, Wicker RB. Materials Science and Engineering a-Structural Materials Properties Microstructure and Processing 2010;527:1861.

[7] Murr LE, Gaytan SM, Martinez E, Medina F, Wicker RB. International journal of biomaterials 2012;2012:245727.

[8] Hernandez-Nava E, Smith CJ, Derguti F, Tammas-Williams S, Leonard F, Withers PJ, Todd I, Goodall R. Acta Materialia 2015;85:387.

[9] Murr LE, Gaytan SM, Medina F, Lopez H, Martinez E, Machado BI, Hernandez DH, Martinez L, Lopez MI, Wicker RB, Bracke J. Philosophical Transactions of the Royal Society a-Mathematical Physical and Engineering Sciences 2010;368:1999.

[10] Fukuda A, Takemoto M, Saito T, Fujibayashi S, Neo M, Pattanayak DK, Matsushita T, Sasaki K, Nishida N, Kokubo T, Nakamura T. Acta Biomaterialia 2011;7:2327.

[11] Heinl P, Mueller L, Koerner C, Singer RF, Mueller FA. Acta Biomaterialia 2008;4:1536.

[12] Salou L, Hoornaert A, Louarn G, Layrolle P. Acta Biomaterialia 2015;11:494.

[13] de Formanoir C, Michotte S, Rigo O, Germain L, Godet S. Materials Science and Engineering: A 2016;652:105.

[14] Sun YY, Gulizia S, Oh CH, Fraser D, Leary M, Yang YF, Qian M. Jom 2016;68:791.

[15] Suard M, Martin G, Lhuissier P, Dendievel R, Vignat F, Blandin JJ, Villeneuve F. Additive Manufacturing 2015;8:124.

[16] Edwards P, O'Conner A, Ramulu M. Journal of Manufacturing Science and EngineeringTransactions of the Asme 2013;135.

[17] Uhlmann E, Schmiedel C, Wendler J. 15th Cirp Conference on Modelling of Machining Operations (15th Cmmo) 2015;31:209.

[18] Pyka G, Burakowski A, Kerckhofs G, Moesen M, Van Bael S, Schrooten J, Wevers M. Advanced Engineering Materials 2012;14:363.

[19] Van Bael S, Kerckhofs G, Moesen M, Pyka G, Schrooten J, Kruth JP. Materials Science and Engineering a-Structural Materials Properties Microstructure and Processing 2011;528:7423.

[20] Pyka G, Kerckhofs G, Papantoniou I, Speirs M, Schrooten J, Wevers M. Materials 2013;6:4737.

[21] Longhitano GA, Larosa MA, Munhoz ALJ, Zavaglia CAD, Ierardi MCF. Materials ResearchIbero-American Journal of Materials 2015;18:838.

[22] Dobrzanski LA, Dobrzanska-Danikiewicz AD, Gawel TG, Achtelik-Franczak A. Archives of Metallurgy and Materials 2015;60:2039. 
[23] de Formanoir C, Suard M, Dendievel R, Martin G, Godet S. Additive Manufacturing 2016;11:71.

[24] Deshpande VS, Fleck NA, Ashby MF. Journal of the Mechanics and Physics of Solids 2001;49:1747.

[25] Michel JC, Moulinec H, Suquet P. International Journal for Numerical Methods in Engineering 2001;52:139.

[26] Koerner C, Bauereiss A, Attar E. Modelling and Simulation in Materials Science and Engineering 2013;21. 